\title{
ACTA BIOLÓGICA CATARINENSE: QUATRO ANOS DE PUBLICAÇÕES
}

\author{
Denise M.D.S. Mouga' ${ }^{1}$ João C.F. Melo Junior²; Bruna T. Possamai ${ }^{3}$
}

\author{
Como citar: \\ MOUGA, D. M. D. S.; MELO JUNIOR, J. C.F.; POSSAMAI, B. T. Acta biológica catarinense: quatro anos de \\ publicações. In: ABEC MEETING, 1, 2017, Curitiba. Anais... Curitiba: Associação Brasileira de Editores \\ Científicos, 2017. p. 50-56. \\ http://dx.doi.org/10.21452/abecmeeting.2017.009
}

\begin{abstract}
Resumo: A Acta Biológica Catarinense (ABC), ISSN 2358-3363, periódico científico criado pelo Departamento de Ciências Biológicas da Universidade da Região de Joinville em 2012, teve sua primeira edição em 2014 e atualmente publica três números por ano, totalizando 27 artigos anuais. É um periódico open access, disponível em formato eletrônico e sem custos para os autores, sendo os artigos submetidos a processo de revisão por pares. Foram publicados até o momento oito números, que totalizam 51 artigos e duas notas científicas, de 185 autores e coautores, de 46 instituições/ entidades e 15 estados brasileiros. Foram recebidos, ao longo do período de atuação da $\mathrm{ABC}, 90$ artigos. $\mathrm{O}$ corpo editorial da $\mathrm{ABC}$ conta com 153 avaliadores de 60 instituições, 16 estados e cinco países. Os artigos recebem o Digital Object Identifier (DOI). Os artigos versam principalmente sobre os temas: Botânica, Zoologia e Ecologia. A qualificação da ABC no Web qualis é, para Biodiversidade, Biotecnologia e Ensino, C, Interdisciplinar B4 e Ciências Agrárias, B3. A ABC está indexada nas plataformas Dialnet e Latindex. A equipe editorial da revista participa de eventos na área de editoração cientifica, visando aprimorar a qualidade da revistar e dar visibilidade para a mesma.
\end{abstract}

Palavras - chave: Open access. Periódico científico. Revista científica.

\begin{abstract}
The Acta Biológica Catarinense (ABC), ISSN 2358-3363, a scientific journal created by the Department of Biological Sciences of the University of the Region of Joinville in 2012, had its first edition in 2014 and currently publishes three issues per year, totaling 27 articles per year. It is an open access journal, available in electronic format and free of charge for authors, with articles submitted to a peer review process. Eight issues have been published so far, totaling 51 articles and two scientific notes, of 185 authors and coauthors, from 46 institutions/ entities and 15 Brazilian states. During the ABC period, 90 articles were received. The $\mathrm{ABC}$ editorial board has 153 referees from 60 institutions, 16 states and five countries. Articles are given the DOI (Digital Object Identifier). The articles are mainly about the themes: Botany, Zoology and Ecology. The qualification of $\mathrm{ABC}$ in the Web qualis is, for Biodiversity, Biotechnology and Teaching, C, Interdisciplinary B4 and Agricultural Sciences, B3. ABC is indexed on Dialnet and Latindex platforms. The editorial team of the journal participates in events in the area of scientific publishing, aiming to improve the quality of the journal and give visibility to it.
\end{abstract}

Keywords: Open access. Scientific journal. Scientific magazine.

\section{INTRODUÇÃO}

Em 1992 foi criado o Departamento de Ciências Biológicas da Universidade da Região de Joinville (Univille), Santa Catarina (SC), que, desde então, integra uma crescente qualificação docente e intensa produção acadêmica, tanto na pesquisa quanto na extensão universitária. O departamento é composto atualmente por 35 professores, em sua maioria doutores, que, em grande parte, trabalham com pesquisa, abarcando a realidade ambiental e da biota da região e buscando respostas aos problemas científicos. Havendo a necessidade de haver uma via que pudesse viabilizar a publicação dessas produções científicas voltadas ao meio ambiente, à biodiversidade e demais áreas da Biologia, e também oferecer uma via de 
MOUGA, D. M. D. S.; MELO JUNIOR; J. C.F.; POSSAMAI, B. T.

"Acta biológica catarinense: quatro anos de publicações"

exposição para estudos e pesquisas realizados no mundo acadêmico externo, numa perspectiva de integração com outras academias, se colocou a conveniência da proposta de criação de uma revista científica. Assim o Departamento de Ciências Biológicas da Univille encaminhou o projeto do periódico às instâncias internas da Universidade, sendo o mesmo aprovado no final de 2012, com o título de Acta Biológica Catarinense (ABC). A ABC tem como objetivo geral contribuir com a divulgação do conhecimento científico voltado para a diversidade biológica no âmbito das Ciências Biológicas, tendo como objetivos específicos agregar trabalhos científicos originais que ampliem o conhecimento sobre a biodiversidade brasileira e mundial, socializar informações que possam ser empregadas em ações de conservação da diversidade biológica retratada nos artigos veiculados pela revista e difundir o conhecimento científico de forma ágil e socialmente responsável. Visando empreender o acompanhamento da evolução da $\mathrm{ABC}$ nos seus primeiros anos de funcionamento, foi realizado o levantamento dos dados referentes à sua progressão, com o propósito de compor um registro histórico e auxiliar o entendimento de seu desenvolvimento.

\section{METODOLOGIA}

Foram realizados levantamentos no histórico da $\mathrm{ABC}$, através de inventários nos documentos do periódico, desde as primeiras atas de reuniões, correspondência trocada entre os envolvidos, arquivos de informações sobre a produção científica recebida e composição dos números publicados. Com base nos mesmos, foram levantados dados tais como o número de artigos recebidos, os autores, os pareceristas, a procedência dos componentes, os comitês integrantes da $\mathrm{ABC}$, a produção revisional dos avaliadores, as áreas de competência dos revisores científicos, as áreas de conhecimento de pertencimento dos artigos e os procedimentos adotados pelo periódico, dentre outros elementos. Estas informações, transformadas em bases numéricas, se encontram tabuladas em banco de dados eletrônico, e são atualizadas regularmente. As informações pesquisadas se referem ao período que vai de 2012 a 2017.

\section{RESULTADOS E DISCUSSÃO}

Caracterização da Revista

As atividades da ABC principiaram no inicio de 2013 e, atualmente, a revista tem suas instalações no Bloco C, sala 321-Z, da Univille, em Joinville, Santa Catarina. Desenvolve 
MOUGA, D. M. D. S.; MELO JUNIOR; J. C.F.; POSSAMAI, B. T.

"Acta biológica catarinense: quatro anos de publicações"

expediente três vezes por semana, no período vespertino. O periódico é de acesso aberto, desobrigado de ônus para os autores. A ABC utiliza a plataforma do Sistema Eletrônico de Editoração de Revistas (SEER) customizado pelo Instituto Brasileiro de Informação, Ciência e Tecnologia (IBICT), software desenvolvido para a construção e gestão de publicação periódica eletrônica, onde a página é mantida online ${ }^{l}$. Possui o identificador de publicações seriadas internacional ISSN 2358-3363. São aceitos manuscritos do campo das Ciências Biológicas, nas áreas correlatas de Aquicultura, Bioarqueologia, Biogeografia, Biologia Da Conservação, Biologia Molecular, Botânica, Ecologia, Ecotoxicologia, Educação Ambiental, Etologia, Evolução, Ficologia, Genética De Conservação, Limnologia, Liquenologia, Micologia, Microbiologia Ambiental, Paleontologia, Palinologia e Zoologia. Apresenta também seção destinada a artigos sobre ensino de biologia. Para possíveis publicações, estão disponíveis, no site da $\mathrm{ABC}$, diretrizes para submissões e desenvolvimento dos trabalhos. Todo o processo de submissão, envio e recebimento de pareceres e reenvio de manuscritos revisados pós avaliação, é realizado em meio eletrônico. Os artigos são revisados por pares (peer review), respeitando a área de conhecimento. Em caso de dupla opinião dos revisores, o manuscrito é avaliado por um terceiro revisor. Todos os avaliadores ad hoc são portadores do título de Doutor ou Mestre e os mesmos não recebem remuneração para avaliar os artigos. A equipe editorial da $\mathrm{ABC}$ é composta por um Editor-chefe e um Editor-assistente, ambos da Univille e uma estagiária. Fazem parte da ABC, também, o Conselho Editorial, composto por oito professores das instituições: Universidade Federal do Paraná (UFPR) (2), Empresa Brasileira de Pesquisa Agropecuária (EMBRAPA) (1), Universidade do Estado do Amazona (UEA) (1), Universidade Regional de Blumenau (FURB) (1), Universidade Federal da Bahia (UFBA) (1), Jardim Botânico do Rio de Janeiro (JBRJ) (1), Universidade de São Paulo (USP) (1) assim como um corpo de Editores de Seção, composto atualmente por 16 especialistas das mais diversas áreas da Biologia (Figura 1). Compõem o corpo de avaliadores da ABC 153 pesquisadores, sendo que 106 atuaram na análise dos artigos submetidos e, dentre eles, 20 pertencem a instituições de ensino superior federais, 8 estaduais, 19 particulares e 11 de outras instituições nacionais ou de outros países. Os revisores que integram a $\mathrm{ABC}$ se situam em diversos estados do Brasil, a saber, Santa Catarina (39), Paraná (23), Rio Grande do Sul (20), São Paulo (19), Rio de Janeiro (16), Minas Gerais (8), Pará (5), Pernambuco (5), Maranhão (4), Amazonas (3), Espirito Santo (3), Mato Grosso do Sul (2), Distrito Federal (2), Paraíba (2), Bahia (1), Piauí (1) e em outros países tais como Canadá (1), Portugal (1) e Suécia (1) (Figura 2). Suas áreas de competência se incluem na Botânica (54), Zoologia (18), Ecologia (13), Educação (8), Microbiologia (1) e outras (11) (Figura 2).

\footnotetext{
${ }^{1}$ http://periodicos.univille.br/index.php/ABC
} 


\section{PERFORMANCE DA REVISTA}

A ABC publicou seu primeiro número em 2014. Até 2016 publicou dois números por ano, em formato eletrônico e, a partir de 2017, passou a publicar três números por ano, com nove artigos por número. Até meados de 2017, oito números foram lançados (Figura 3). Nos quase cinco anos de atuação da $\mathrm{ABC}$, foram recebidos 90 artigos científicos. Destes, nove foram recusados por dois ou mais avaliadores, um não se enquadrou no escopo da revista, três desistiram de publicar, sete autores não responderam mais, 53 foram aceitos e 16 estão atualmente em processo de revisão (Figura 4). Os artigos provêm de 185 autores e coautores, de 15 instituições de ensino estaduais, 21 federais, 40 particulares e 10 de outras instituições, pertencentes aos estados de Santa Catarina (43), São Paulo (13), Paraná (7), Rio Grande do Sul (5), Rondônia (4), Minas Gerais (4), Amazonas (3), Espirito Santo (2), Distrito Federal (2), Pará (2), Rio de Janeiro (1), Mato Grosso do Sul (1), Bahia (1) e Tocantins (1).

Figura 1: Quantidade de integrantes dos comitês da ABC por estado do Brasil.

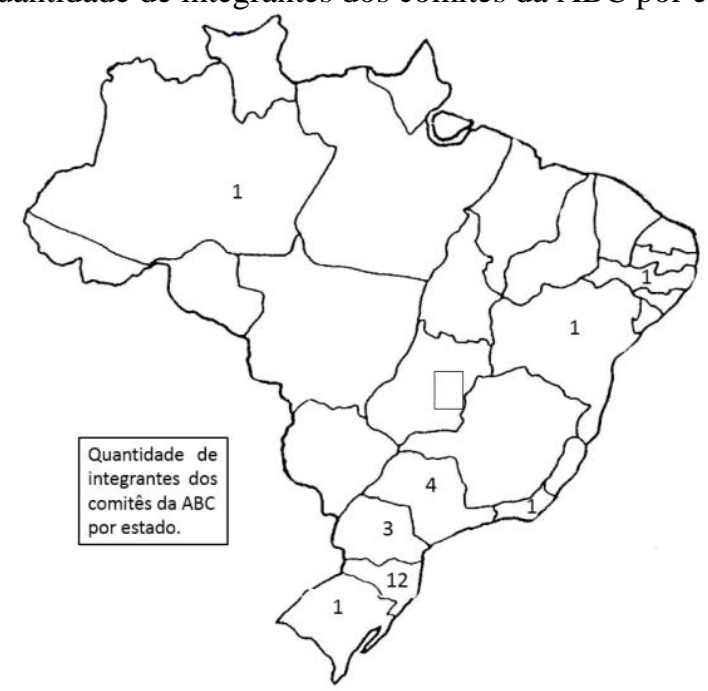


MOUGA, D. M. D. S.; MELO JUNIOR; J. C.F.; POSSAMAI, B. T.

"Acta biológica catarinense: quatro anos de publicações"

Figura 2: Quantidade de revisores por estados do Brasil.

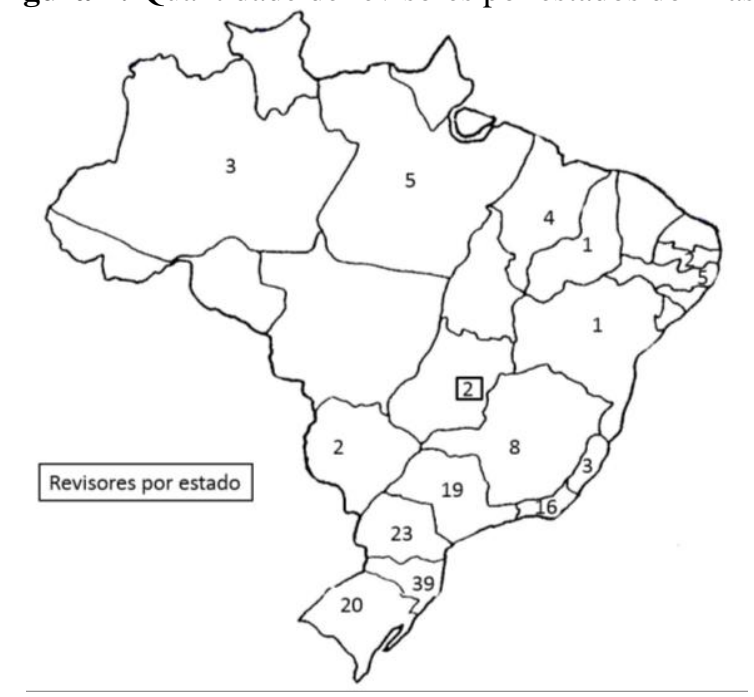

Figura 3: As capas dos números publicados da $\mathrm{ABC}$.
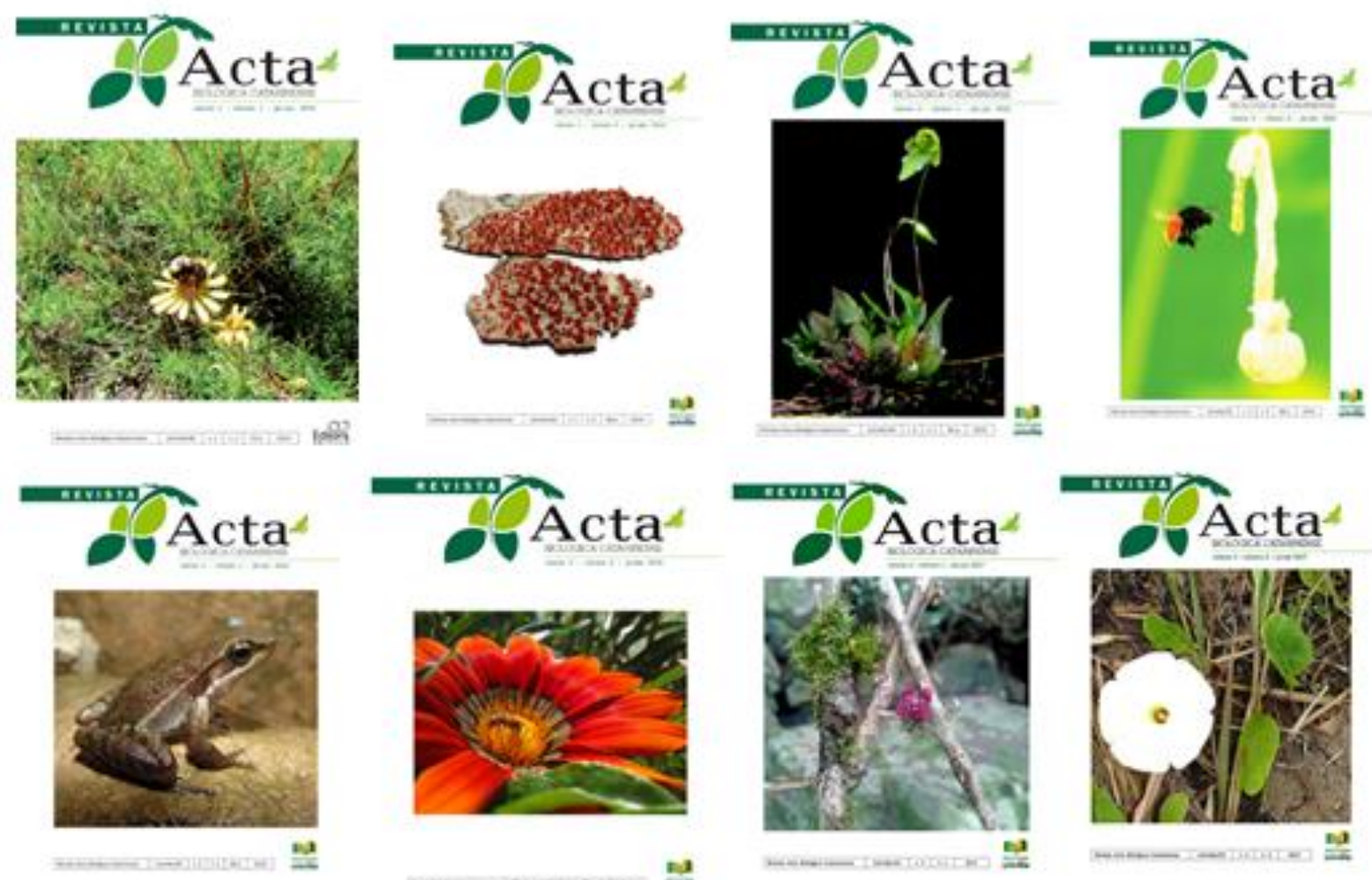

Os artigos publicados se encaixam principalmente nas áreas de Botânica (34 artigos), Zoologia (12) e Ecologia (6). Os artigos recebem o DOI (Digital Object Identifier), que foi integrado a todos os artigos já publicados pela ABC. As normas de publicação para a submissão de manuscritos têm sido atualizadas e melhoradas, estando já na sua $11^{\circ}$ edição, visando uma padronização e sistematização da forma. Dentre estas normas, destaca-se aquela que incentiva a redação dos artigos em língua inglesa, almejando a internacionalização do periódico e seu maior alcance nas esferas científicas. 
Figura 4 - Proveniência dos artigos por estado do Brasil.

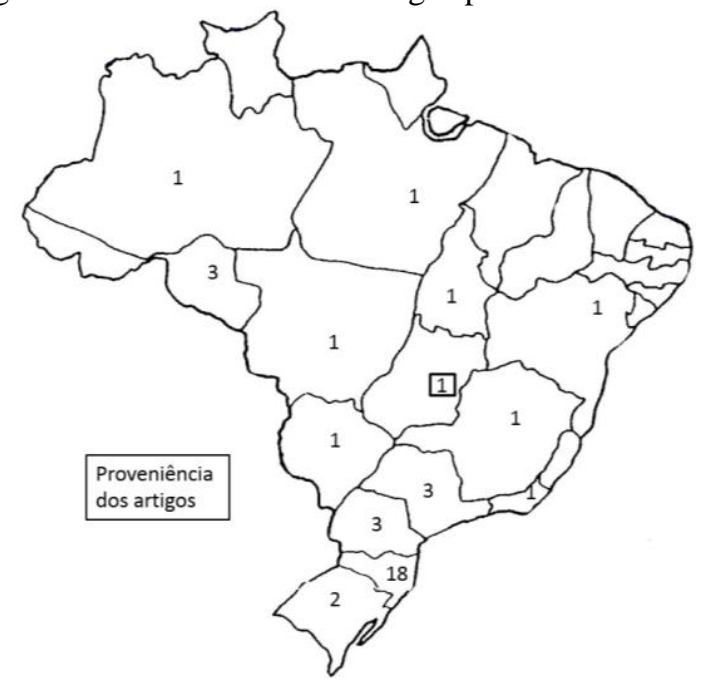

A equipe editorial tem participado de eventos de editoria cientifica tais como ABEC Meeting 2017, 23 Curso de Editoração Científica, XV ENEC - Encontro Nacional de Editores Científicos, I Evento Regional SSP e I Encontro de Editores e Consultores de Revistas Cientificas, com o objetivo de dar visibilidade à $\mathrm{ABC}$. A ABC encontra-se indexada nas plataformas Dialnet e Latindex e está se adequando às normas de SciElo, Web of Science, SCOPUS, PubMED e RedALyC, plataformas indicadas pelo CNPq para apoiar propostas de editoração e publicação de periódicos científicos brasileiros de alta especialização, em todas as áreas de conhecimento. A ABC visa também representar a produção intelectual do programa de pós-graduação a nível de Mestrado, a ser implantado na Univille, na área de Ciências Ambientais, fruto da experiência de 25 anos de funcionamento do curso de graduação em Ciências Biológicas. Dentre as medidas visando incrementar a qualidade da publicação, a ABC pretende instalar um contador de acessos para mensurar sua visibilidade, manter a periodicidade de três números por ano com nove artigos por número e melhorar a qualidade e a diversidade dos artigos publicados. No Programa Web qualis, a $A B C$, na sua primeira avaliação quadrienal, recebeu as seguintes classificações, pelas áreas de avaliação biodiversidade, biotecnologia e ensino: classificação C, interdisciplinar: B4 e ciências agrárias: B3 (PLATAFORMA SUCUPIRA, 2016). A classificação alcançada tem servido como balizador das ações da $\mathrm{ABC}$ e servirá para aperfeiçoar as metas a serem atingidas quando dos processos de avaliação dos Programas de Pós-Graduação stricto sensu pois pondera a qualidade da produção intelectual dos docentes e pesquisadores. Deste modo, a $\mathrm{ABC}$ vem cumprindo seu papel de divulgação do conhecimento científico na área das Ciências Biológicas, em termos nacionais. 
"Acta biológica catarinense: quatro anos de publicações"

\section{REFERÊNCIAS}

PLATAFORMA SUCUPIRA. Qualis Periódicos. Ministério da Educação. UFRN. Brasília; 2016. Disponível em:

$<$ https://sucupira.capes.gov.br/sucupira/public/consultas/coleta/veiculoPublicacaoQualis/lista ConsultaGeralPeriodicos.jsf >. Acesso em: 25 out. 2017. 